\title{
Implementing Truthful Routing Path Generation in WSN through TARF
}

\author{
Premakshi B.Dohe \\ MTech Scholor \\ Department of Computer Science and \\ Engineering \\ Sagar Institute of Research and Technology, \\ Bhopal
}

\author{
Rajesh K Shukla \\ Head, Department of Computer Science and \\ Engineering \\ Sagar Institute of Research and Technology, \\ Bhopal
}

\begin{abstract}
Wireless device networks are susceptible to a good set of security attacks, together with those targeting the routing protocol practicality. The multi-hop routing in wireless device networks (WSNs) offers very little protection against identity deception through replaying routing data. Associate in Nursing opposer will exploit this defect to launch numerous harmful or perhaps devastating attacks against the routing protocols, together with sink attacks, hollow attacks and Sybil attacks. To face this downside, we tend to propose a truthful routing protocol that adopts the routing principle to address the network dimensions, and depends on a distributed trust model for the detection and dodging of malicious neighbors. true is any aggravated by mobile and harsh network conditions. ancient cryptographical techniques or efforts at developing trust-aware routing protocols don't effectively address this severe downside. Further, we've enforced allow-overhead TARF module in TinyOS; as incontestable, this implementation may be incorporated into existing routing protocols with the smallest amount effort. supported TARF, we tend to additionally incontestable a proof-of-concept mobile target detection application that functions well against Associate in Nursing anti-detection mechanism.

To secure the WSNs against adversaries misdirecting the multi-hop routing, we've designed and enforced TARF, a strong trust-aware routing framework for dynamic WSNs.
\end{abstract}

Keywords: control overhead, dropping ratio, delay energy efficient routing, jitter, secure routing, sensor energy, simulation throughput,trust-aware routing, WSN.

\section{INTRODUCTION}

Wireless device networks (WSNs) ar ideal candidates for applications to report detected events of interest, like military police work and fire observation. A WSN includes powered man nodes with extraordinarily restricted process capabilities. With a slim radio communication vary, a device node wirelessly sends messages to a base station via a multihop path. However, the multi-hop routing of WSNs usually becomes the target of malicious attacks. Associate in Nursing assaulter might tamper nodes physically, produce traffic collision with apparently valid transmission, drop or misdirect messages in routes, or jam the communication by making radio interference. This paper focuses on the sort of attacks within which adversaries misdirect network traffic by identity deception through replaying routing data. supported identity deception, the opposer is capable of launching harmful and hard-to-detect attacks against routing, like selective forwarding, hollow attacks, sink attacks and Sybil attacks.

Wireless device Networks (WSNs) supply economical, cheap solutions for a good kind of application domains together with military fields, healthcare, independent agency, business management, intelligent inexperienced aircrafts and control in sensible roads. Wireless device network consists of a robust base station and a collection of low-end device nodes. Base station and device nodes have wireless capabilities and communicate through a wireless, multi-hop, ad-hoc network.[3]Wireless device networks (WSN) have emerged as a vital new technology for instrumenting and perceptive the physical world.

Although networking and security technologies ar in a very mature stage, the restricted device node resources in terms of memory house, process power and energy availableness, constrain the complexness of the safety mechanisms which will be enforced, dictating the necessity for brand new protocol approaches style. WIRELESS device networks (WSNs) ar a capable situation for sensing giant areas at high abstraction and positive resolution. However, the little size and low value of the process machines that produces them enticing for giant preparation additionally causes the loss of low operational reliability[1]. As a harmful and easy-toimplement style of attack, a malicious node merely replays all the outgoing routing packets from a legitimate node to forge the latter node's identity; the malicious node then uses this cast identity to participate within the network routing, so disrupting the network traffic. Wireless device networks (WSN) have emerged as a vital new technology for instrumenting and perceptive the physical world. the essential building block of those networks may be a little micro chip integrated with one or additional MEMS (microelectromechanical system) sensors, actuators, and a wireless transceiver.[2] A WSN is sometimes assortment of tons of or thousands of device nodes.

These device nodes ar usually densely deployed in a very device field and have the power to assemble knowledge and route knowledge back to a base station (BS). A device has four basic parts: a sensing unit, a process unit, a transceiver unit, and an influence unit [5]. Most of the device network routing techniques and sensing tasks need information of location, that is provided by a location finding system. Wireless device network contains sizable amount of nodes every|and every\} node is also terribly near each neighbor. Since WSN ought to use multihop techniques as a result of it consume less power than single hop techniques.

Multihop techniques may effectively overcome a number of the signal propagation outcomes skilled in long-distance wireless communication [6]. WSN extraly |might also|may additionally\} have additional application dependent elements like a location finding, system, power generator, and mobilizer (Fig. 1). This same technique may be used to conduct another sturdy kind of attack - Sybil attack: through 
replaying the routing data of multiple legitimate nodes, Associate in Nursing assaulter might gift multiple identities to the network. a legitimate node, if compromised, may launch of these attacks. a poor network association causes a lot of problem in identifying between Associate in Nursing assaulter and a honest node with transient failure. while not correct protection, WSNs with existing routing protocols may be fully destroyed beneath bound circumstances. In Associate in Nursing emerging sensing application through WSNs, saving the network from being destroyed becomes crucial to the success of the appliance. Sensing units ar typically composed of 2 sub units: sensors and analog-todigital converters (ADCs).

As so much as WSNs ar involved, secure routing solutions supported trust and name management seldom address the identity deception through replaying routing data.The countermeasures planned to this point powerfully depends on either tight time synchronization or identified geographic data whereas their effectiveness against attacks exploiting the replay of routing data has not been examined nonetheless. At this time, to guard WSNs from the harmful attacks exploiting the replay of routing data, we've designed and enforced a strong trust-aware routing framework, TARF, to secure routing solutions in wireless device networks.

\subsection{Options OF Wsn}

The vital options of a WSN embody

- Limited Power consumption for nodes victimization batteries or energy gathering

- Ability to run with node failures

- Mobility of nodes

- Dynamic configuration

- Communication failures

- Heterogeneity of nodes

- Scalability to giant scale of exploitation

- capacity to survive exhausting environmental conditions

- Easy to use

- Unattended operation

- Power consumption

As WSNs ar ample like ancient wireless unintentional networks, vital variations exist that greatly influence however security is achieved [4]. In [8], I. F. Akyildiz et al planned the variations between device networks and unintentional networks are:

1. the amount of device nodes in a very device network may be many orders of magnitude on top of the nodes in a poster hoc network.

2. device nodes ar densely deployed.

3. device nodes ar lying face right down to failures attributable to harsh environments and energy constraints.

4. The topology of a device network changes terribly ofttimes attributable to failures or quality.

5. device nodes ar restricted in computation, memory, and power resources.

6. device nodes might not have international identification.

Authentication necessities

Though a particular application might verify whether or not encoding is required, TARF needs that the packets ar properly attested, particularly the published packets from the bottom station. the published from the bottom station is unsymmetrically attested therefore on guarantee that Associate in Nursing opposer isn't ready to manipulate or forge a broadcast message from the bottom station at can. significantly, with attested broadcast, even with the existence of attackers, TARF might use Trust Manager and therefore the received broadcast packets regarding delivery data to decide on trustworthy path by circumventing compromised nodes. while not having the ability to physically capturing the bottom station, it's typically terribly tough for the opposer to control the bottom station broadcast packets that ar unsymmetrically attested. The uneven authentication of these broadcast packets from the bottom station is crucial to any undefeated secure routing protocol. It may be achieved through existing unsymmetrically attested broadcast schemes which will need loose time synchronization. As Associate in Nursing example, $\mu$ TESLA achieves uneven attested broadcast through a cruciate cryptographical formula and a loose delay schedule to disclose the keys from a key chain. alternative samples of uneven attested broadcast schemes requiring either loose or no time synchronization are found. Considering the good computation value incurred by a robust uneven authentication theme and therefore the problem in key management, a daily packet apart from a base station broadcast packet might solely be moderately attested through existing cruciate schemes with a restricted set of keys, like the message authentication code provided by TinySec. it's potential that Associate in Nursing opposer physically captures a non-base legal node and divulges its key for the cruciate authentication. thereupon key, the opposer will forge the identity of that non-base legal node and joins the network "legally". However, once the opposer uses its faux identity to incorrectly attract a good quantity of traffic, once receiving broadcast packets regarding delivery data, alternative legal nodes that directly or indirectly forwards packets through it'll begin to pick out a additional trustworthy path through Trust Manager.

Wireless device networks (WSNs) ar ideal candidates for applications to report detected events of interest, like military police work and fire observation. A WSN includes powered man nodes with extraordinarily restricted process capabilities. With a slim radio communication vary, a device node wirelessly sends messages to a base station via a multihop path. However, the multi-hop routing of WSNs usually becomes the target of malicious attacks. Associate in Nursing assaulter might tamper nodes physically, produce traffic collision with apparently valid transmission, drop or misdirect messages in routes, or jam the communication by making radio interference. various security attacks are conferred within the literature ([6], [7]) with a big set targeting the routing method [8]. Once Associate in Nursing opposer node manages to participate within the network, it will harm the routing method by merely dropping the packets it receives for forwarding, i.e. denying to sincerely work within the routing procedure. Another simply implementable attack is packet modification. A taxonomy of routing attacks may be found in [9].

To defend against the bulk of routing attacks, Associate in Nursing approach borrowed from the human society has been planned [10]: nodes monitor the behavior of their neighbors so as to judge their trait, relating to specific behaviour aspects referred to as trust metrics.

Although a embarrassment of such models has been planned and shown to with efficiency mitigate routing attacks, trust models ar themselves susceptible to specific attacks [11]. the necessity to defend against these attacks any will increase the complexness of the practicality that has to be enforced on the device nodes for security functions.

As a harmful and easy-to-implement style of attack, a malicious node merely replays all the outgoing routing 
packets from a legitimate node to forge the latter node's identity; the malicious node then uses this cast identity to participate within the network routing, so disrupting the network traffic. Those routing packets, together with their original headers ar replayed with none modification. notwithstanding this malicious node cannot directly hear the valid node's wireless transmission, it will conspire with alternative malicious nodes to receive those routing packets and replay them somewhere far-flung from the first valid node, that is understood as a hollow attack. Since a node in a very WSN typically depends alone on the packets received to understand regarding the sender's identity, replaying routing packets permits the malicious node to forge the identity of this valid node. once "stealing" that valid identity, this malicious node is in a position to misdirect the network traffic. as an example, it should drop packets received, forward packets to a different node not imagined to be within the routing path, or perhaps type a transmission loop through that packets ar passed among a number of malicious nodes infinitely. it's usually tough to understand whether or not a node forwards received packets properly even with overhearing techniques. sink attacks ar another quite attacks which will be launched once stealing a legitimate identity. in a very sink attack, a malicious node might claim itself to be a base station through replaying all the packets from a true base station. Such a faux base station may lure quite 0.5 the traffic, making a "black hole". This same technique may be used to conduct another sturdy kind of attack - Sybil attack: through replaying the routing data of multiple legitimate nodes, Associate in Nursing assaulter might gift multiple identities to the network. a legitimate node, if compromised, may launch of these attacks. a poor network association causes a lot of problem in identifying between Associate in Nursing assaulter and a honest node with transient failure. while not correct protection, WSNs with existing routing protocols may be fully destroyed beneath bound circumstances. In Associate in Nursing emerging sensing application through WSNs, saving the network from being destroyed becomes crucial to the success of the appliance. sadly, most existing routing protocols for WSNs each assume the honesty of nodes and specialise in energy potency, or arrange to exclude unauthorized participation by encrypting knowledge and authenticating packets. samples of these secret writing and authentication schemes for WSNs embody TinySec, Spins, TinyPK, and TinyECC. Admittedly, it's vital to contemplate economical energy use or battery power-driven device nodes and therefore the strength of routing beneath topological changes further as common faults in a very wild surroundings.However, it's additionally vital to include security mutually of the foremost vital goals; meantime, even with excellent secret writing and authentication, by replaying routing data, a malicious node will still participate within the network victimization another valid node's identity. The gossiping-based routing protocols supply bound protection against attackers by choosing random to forward packets, however at a worth of substantial overhead in propagation time and energy use. neighbors additionally to the cryptographical ways, trust and name management has been used in generic unintentional networks and WSNs to secure routing protocols. Basically, a system of trust and name management assigns every node a trust price in keeping with its past performance in routing. Then such trust values are wont to facilitate decide a secure and economica route. However, the planned trust and name management systems for generic unintentional networks target solely comparatively powerful hardware platforms like laptops and sensible phones. Those systems can't be applied to WSNs attributable to the excessive overhead for resourceconstrained device nodes power-driven by batteries.

\subsection{Consideration}

In a knowledge assortment task, a device node sends its sampled knowledge to an overseas base station with the help of alternative intermediate nodes, as shown in Figure one. although there may be quite one base station, our routing approach isn't full of the amount of base stations; to change our discussion, we tend to assume that there's only 1 base station. Associate in Nursing opposer might forge the identity of any legal node through replaying that node's outgoing routing packets and spoofing the acknowledgement packets, even remotely through a hollow.

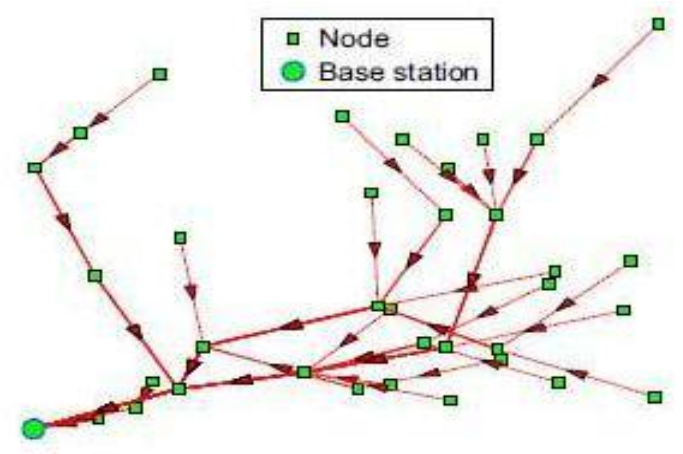

Fig.1 Multi hop routing

Nonetheless, our approach will still be applied to cluster based mostly WSNs with static clusters, wherever knowledge are collective by clusters before being relayed. Cluster-based WSNs permits for the good savings of energy and information measure through aggregating knowledge from kids nodes and playacting routing and transmission for kids nodes. in a very cluster-based WSN, the cluster headers themselves type a sub-network; once bound knowledge reach a cluster header, the collective knowledge are routed to a base station solely through such a sub-network consisting of the cluster headers. Our framework will then be applied to the present sub-network to attain secure routing for cluster based mostly WSNs. TARF might run on cluster headers solely and therefore the cluster headers communicate with their kids nodes directly since a static cluster has identified relationship between a cluster header and its kids nodes, although any link-level safety features is also any used. Finally, we tend to assume an information packet has a minimum of the subsequent fields: the sender id, the sender sequence variety, the next-hop node id (the receiver during this one hop transmission), the supply id (the node that initiates the data), and therefore the source's sequence variety. we tend to insist that the supply node's data ought to be enclosed for the subsequent reasons as a result of that permits the bottom station to trace whether or not an information packet is delivered. it'd cause an excessive amount of overhead to transmit all the one hop data to the bottom station. Also, we tend to assume the routing packet is sequenced.

\subsection{Goals}

TARF primarily guards a WSN against the attacks misdirecting the multi-hop routing, particularly those supported fraud through replaying the routing data. This paper doesn't address the denial-of-service (DoS) attacks, wherever Associate in Nursing assaulter intends to wreck the 
network by exhausting its resource. as an example, we tend to don't address the DoS attack of congesting the network by replaying various packets or physically jam the network. TARF aims to attain the subsequent fascinating properties:

High turnout- Throughput is outlined because the quantitative relation of the amount of all knowledge packets delivered to the bottom station to the amount of all sampled knowledge packets. Through place reflects however with efficiency the network is grouping and delivering knowledge. Here we tend to regard high turnout mutually of our most vital goals.

Energy Efficiency - knowledge transmission accounts for a significant Portion of the energy consumption. we tend to measure energy potency by the typical energy value to with success deliver a unit-sized knowledge packet from a supply node to the bottom station. lean enough attention once considering energy value since every re-transmission causes an understandable increase in energy consumption. If each node in a very WSN consumes or so constant energy to transmit a unit-sized knowledge packet, we are able to use another metric hop-per-delivery to judge energy potency. thereunder assumption, the energy consumption depends on the amount of hops, i.e. the amount of one-hop transmissions occurring. to judge however with efficiency energy is employed, we are able to live the typical hops that every delivery of an information packet takes, abbreviated as hopper-delivery.

Scalability \& Adaptability - TARF ought to work well with WSNs of huge magnitude beneath extremely dynamic contexts. we are going to measure the quantifiability and flexibility of TARF through experiments with large-scale WSNs and beneath mobile and hash network conditions.

\subsection{Style Of Tarf}

The design of energy-efficient routing protocols in WSNs is influenced by several factors. These factors should pass though before economical communication may be achieved in WSNs.

Here may be a list of the foremost common factors poignant the routing protocols design:

- Node Deployment: it's Associate in Nursing applicationdependent operation poignant the routing

protocol performance, and may be either settled or irregular. - Node/Link Heterogeneity: The existence of heterogeneous set of sensors offers rise to several technical issues associated with knowledge routing and that they ought to be overcome

- knowledge coverage Model: knowledge sensing, measuring and coverage in WSNs rely upon the appliance and therefore the time criticality of the information coverage. knowledge coverage may be classified as either time-driven (continuous), event driven, query-driven, or hybrid.

- Energy Consumption while not Losing Accuracy:

In this case, energy-conserving mechanisms of information communication and process ar quite necessary.

- Scalability: WSNs routing protocols ought to be climbable enough to retort to events, e.g. Brobdingnagian increase of device nodes, within the surroundings.

- Network Dynamics: quality of device nodes is critical in several applications; despite the actual fact that almost all of the network architectures assume that device nodes ar stationary.
- Fault Tolerance: the general task of the device network shouldn't be full of the failure of device nodes.

- Connectivity: The device nodes property depends on the random distribution of nodes.

- Transmission Media: in a very multi-hop WSN, act nodes ar connected by a wireless medium. One approach of macintosh style for device networks is to use TDMA based mostly protocols that conserve additional energy compared to contention-based protocols like CSMA (e.g., IEEE 802.11)

- Coverage: In WSNs, a given sensor's read of the surroundings is proscribed each in vary and in accuracy;

- Quality of Service: knowledge ought to be delivered among a precise amount of your time. However, in a very sensible variety of applications, conservation of energy, that is directly associated with network life, is taken into account comparatively additional vital than the standard of information sent. Hence, energy aware routing protocols ar needed to capture this demand.

- knowledge Aggregation: knowledge aggregation is that the combination of information from totally different sources in keeping with a precise aggregation operate, e.g. duplicate suppression.

All the on top of factors ar mentioned thoroughly in [1].

Routing in WSNs is extremely difficult attributable to the inherent characteristics that distinguish these networks from alternative wireless networks like mobile unintentional networks or cellular networks. Following ar the challenges and style problems for implementing routing protocols in WSN than alternative style of networks.

\section{IMPLEMENTATION}

Consider a network depicted as a graph $\mathrm{G}(\mathrm{N} ; \mathrm{L})$, wherever $\mathrm{N}$ and $\mathrm{L}$ denote a collection of nodes and adrift links, severally. The nodes ar numbered from one through $\mathrm{jN} \mathrm{j}$. A link ' a pair of $\mathrm{L}$ is assumed to be bi-directional. Let $\mathrm{x}^{`}$ and $\mathrm{y}$ ' denote the identifiers of the nodes connected by link specified $x^{`}<y^{`}$.

Let A represent the set of directional links, or arcs, within the network. Associate in Nursing arc from node $i$ to $j$ is denoted as $(i ; j)$.

The failure of link `is assumed to have an effect on the arcs in each directions. Let $\mathrm{F}$ denote the set of dual-link failures to be tolerated. a part $\mathrm{f}$ a pair of $\mathrm{F}$ consists of specifically 2 adrift links, or correspondingly four directed arcs.

A. spare condition for existence of an answer Three-edge-connectivity may be a necessary condition for a network to be resilient to dual-link failures. it's additionally spare that a network is three-edge-connected so as to get a solution for BLME downside, evidenced as follows.

Assume that the given network is split into $\mathrm{jLj}$ auxiliary graphs. Associate in Nursing auxiliary graph $\mathrm{X}^{\prime}$ is built by removing link ` from the first network: $X^{`}=G\left(N ; L \square f^{\prime} g\right)$. In every auxiliary graph $X^{\prime}$, the goal is to spot a path $\mathrm{P}^{\prime}$ from node $x `$ to $y$. Let $-" 0$ be a binary variable that indicates whether or not link 0 is gift within the backup path of link `: one if true, 0

otherwise.

Let $\square$ be the mathematician operate that denotes the property between nodes $x^{\prime}$ and $y^{\prime}$ within the auxiliary graph $X$, depicted as a operate of the variable set $f_{-}{ }^{\prime} " 0$ : $` 0$ a pair of $X^{\prime} \mathrm{g}$.

Consider the instance network shown in Figure 5(a). The auxiliary graph such as link one is shown in Figure 5(b). The mathematician operate representing the property between nodes A and B is shown in Equations (1) and (2) in Sum-of- 
Product and Product-of-Sum forms, severally.

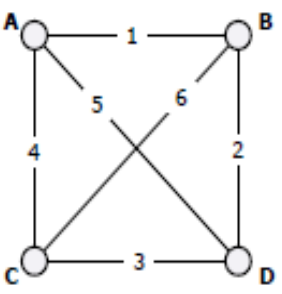

(a)

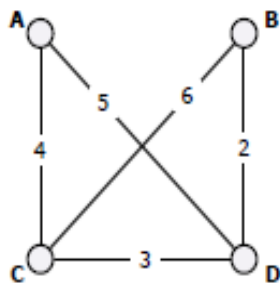

(b)
Fig:2 (a)Example Network (b)Network after failure at link 1

Minimizing spare capability

If the target is to reduce the spare capability allotted within the network, then links is also faraway from the given network till the reworked network simply meets the required conditions for the existence of an answer. For a given network $G(N ; L)$, let Sij denote the amount of link-disjoint methods between the nodes $i$ and $j$. If there exists quite 3 link-disjoint methods, then $\mathrm{Sij}$ is truncated to a few, as threeedge-connectivity may be a spare condition for the existence of an answer to the BLME downside. Let $\mathrm{G} 0(\mathrm{~N} ; \mathrm{LO})$ denote the reworked graph, wherever L0 nine L, specified the property between all node pairs is maintained in graph G0. Given G0, the spare capability on a link ' 0 a pair of L0 is also computed as follows:

(1) if a link is needed to keep up three-connectivity between 2 node pairs in G0, then it needs 2 spare fibers;

(2) if a link is needed to keep up 2-connectivity between two node pairs, then it needs one spare fiber;

(3) if a link is needed to keep up (one-)connectivity between 2 node pairs, it doesn't need any spare fibers. The links faraway from the given network needn't be equipped with spare fibers.

If a given network is three-edge-connected, then the target is to stay the graph three-edge-connected with the minimum variety of links, that may be a well-known to be NPHard.

In such a reduced network, each link can use 2 spare fibers. once a network is a smaller amount than three-edge connected, then a link ' should need 2 spare fibers despite the fact that a dual-link failure involving ' might disconnect the graph. as an example, contemplate the NJ-LATA network shown in Figure 10(f). There ar 3 dual-link failures that disconnect the graph. However, links one \{and a pair of|and a couple of|and a pair of $\}$ ar needed to keep up threeconnectivity between nodes 2 and three. Hence, 2 spare fibers ar needed on links one and a couple of. On the opposite hand, the removal of 1 of the opposite four links $(11,16,22$, and 23) doesn't violate the three-connectivity of alternative nodes. Hence, links 11, 16, 22, and twenty three is also equipped with only 1 spare fiber as long as \{they ar|they're\} not needed to keep up the property between the node pairs once alternative links are removed. Note that if link twenty is removed, then links twenty two and twenty three ar needed to keep up three-connectivity between nodes nine and ten, thus would force 2 fibers on every. the rise in spare capability needed in alternative links attributable to removal of a link depends on the configuration. it's not necessary that minimizing the spare capability implies minimizing $\mathrm{jLOj}$ in a very graph that's but three-connected (or vice versa). If a network is a minimum of threeconnected, then minimizing spare capability is equivalent of minimizing the amount of links to stay the graph 3 connected.

\subsection{Heuristic Approach}

As ILP resolution times for giant networks is also prohibitively high, a heuristic approach is additionally developed. The heuristic resolution is predicated on repetitious computation of minimum value routing. The network is treated as Associate in Nursing adrift graph G. a collection of auxiliary graphs such as failure of a link 'a pair of $G$ is created: $X^{\prime}=G\left(N ; L \square f^{\prime} g\right)$. In every auxiliary graph $X^{\prime}$, the target is to get a path between the nodes that were originally connected by link `. Let $\mathrm{P}^{\prime}$ denote the trail hand-picked in auxiliary graph $\mathrm{X}$ '. If a link ' 0 may be a a part of the trail hand-picked on graph $X^{\prime}$, then the trail in graph $X^{`} 0$ should avoid the utilization of link `. this is often accomplished by imposing a price on the links within the auxiliary graphs, and having the trail choice approach choose the minimum value path. Let $\mathrm{w}^{\text {" }} 0$ denote the price of link ` 0 on graph $X{ }^{`}$ specified it indicates that graph $X ` 0$ contains link ' and therefore the 2 links ' and ' 0 is also unprocurable at the same time. Hence, the price values ar binary in nature. the price of a path in Associate in Nursing auxiliary graph is that the add of the price of links in it. At any given instant throughout the computation, the overall value of all the methods $(\mathrm{T})$ is that the add of the price of the methods across all auxiliary graphs. it should be ascertained that the overall value should be an excellent variety, as each link ` 0 in a very path $\mathrm{P}^{`}$ that encompasses a value of one implies that link ` in path $\mathrm{P}^{`} 0$ would even have a price of one. For a given network, the minimum price of the overall value would then be two occasions the amount of dual-link failure situations that will have the network disconnected.

Note that the weights of the links within the auxiliary graphs ar initialized to zero (Step a pair of of the IMCP heuristic). The weights is also initialized to atiny low positive price nine to get backup methods with shorter path lengths. Such a modification, however, would end in a trade-off between the typical backup path length and therefore the variety of dual-link failures tolerated.

\subsubsection{Loop formation}

The backup path of a link 'once its failure is analogous to a association established in a very network that is protected victimization link protection (for the second failure). Hence, all the properties of a link protection strategy for a association in a very regular network is valid in dual-link failure resiliency victimization link protection. Loop formation is one amongst them! contemplate a unsuccessful link (connecting nodes one and 8) whose backup is established on a path wherever the nodes within the path are numbered from one through eight. Figure nine shows 2 types of loop formation.

In Figure 9(a), link 4-5 is protected by the backup path 4-76-5. Upon failure of link $4-5$, the backup between nodes one and eight is changed as $1-2-3-4-7-6-5-6-7-8$, leading to the loop 7-6-5-6-7. whereas this loop may be cropped victimization sign, it's solely necessary to cut back path delay. The backup path for $4-5$ can route each its primary fiber and therefore the secondary fiber of link 1-8 through the trail 4-7-6-5, thus 2 spare fibers are utilized in every of those links. The loop formation involves constant links, but the links ar traversed in wrong way. As each link has to be equipped with 2 spare fibers in every direction (for bidirectional connectivity), there'll not be a resource rivalry. 


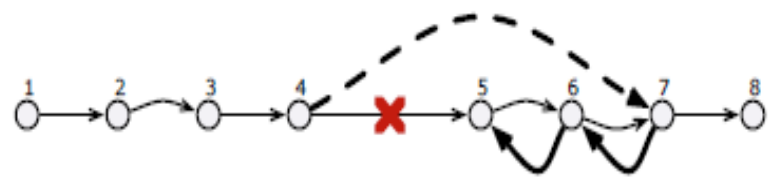

(a) No resource contention

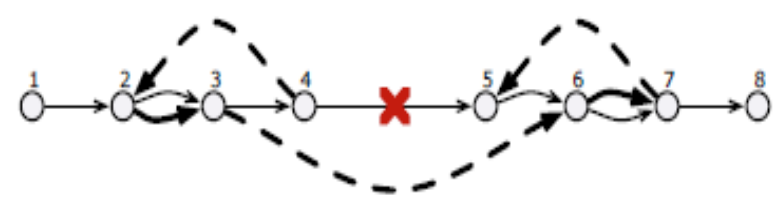

(b) Contention on links traversed in same direction

Figure 3(b) shows another quite loop formation wherever there's a possible resource rivalry. The backup path of link $4-5$ is $4-2-3-6-7-5$. Upon failure of link $4-5$, the backup between nodes one and eight is changed as: $1-2-3-4-2-3-$ 6-7-5-6-7-8; leading to 2 loops $2-3-4-2$ and 7-5-6-7. Note that links $2-3$ and 6-7 ar traversed within the same direction. If such a loop formation is allowed, then links 2-3 and 6-7 should be equipped with 3 spare fibers because the backup path for link 4-5 would be switch 2 fibers. because the network is assumed to own at the most 2 link failures, it should be spare to equip each link with 2 spare fibers. Therefore, if the links have solely 2 spare fibers, pruning of the backup methods can't be avoided. The cropped backup path between nodes one and eight once link 4-5 failure would be 1-2-3-6-7-8; whereas the backup path of link 45 would be $4-2-3-6-7-5$. The process downside delineate here is comparable to those encountered in any link protection mechanism. The methods may be cropped employing a sign mechanism that will be needed to ascertain the backup methods. Note that the sign can't be fully avoided as a link will function backup for quite 2 alternative links, thus protection switches can't be designed before failure.

The use of the cruciate key secret writing is common to confirm knowledge integrity. cruciate key secret writing code may be divided into the block cipher and stream one, and block cipher formula has been developed extensively. Currently, celebrated block cipher algorithms were created by the general public project like AES (Advanced secret writing Standard) project of the us, Loch Ness monster (New European Schemes for Signatures. Integrity, and Encryption) project of Europe, and CRYPTREC (Cryptography analysis and analysis Committees) project of Japan [1]-[3].

In the starting, block cipher formula was primarily enforced via software system, however intensive researches regarding hardware implementation of secret writing and secret writing are dispensed for quick operations. The block cipher may be classified into Feistel structure and SPN (Substitution Permutation Network) one [4]-[5]. Feistel structure has a plus of constant formula between secret writing and secret writing, and therefore the feature of SPN structure is that it's a distinct formula between secret writing and secret writing. specifically, the SPN structure encompasses a disadvantage that its space will increase double compared with the Feistel one once SPN structure is enforced via hardware.

RC6, that may be a straightforward, fast, and secure block cipher, was the ultimate candidate formula within the AES project of the us and therefore the Loch Ness monster project of Europe. These comes need 128-bit and variablelength block cipher secret writing formula. RC6 encompasses a changed Feistel structure and a drawback that it's totally different formula between secret writing and secret writing. Thus, the RC6 formula wants double house compared with constant structure of secret writing and secret writing once it's enforced on hardware. during this paper, we tend to propose Associate in Nursing improved RC6 secret writing formula that have constant structure of secret writing and secret writing. we tend to devise our formula by inserting a cruciate layer victimization straightforward rotation and XOR operations, within which the half whole RC6 rounds uses secret writing procedure and therefore the remainder of it ar employs secret writing one.
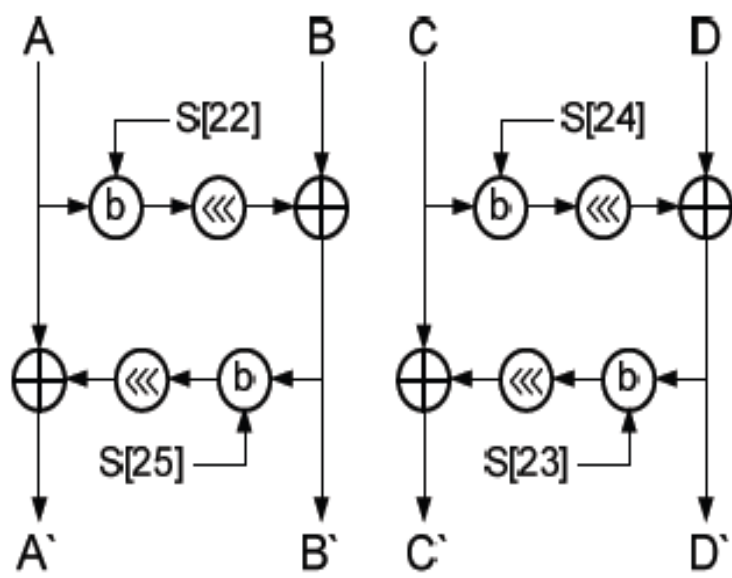

Fig.4 The implementation of symmetry layer in RC6

The symmetry layer is place between secret writing half and secret writing one. The planned RC6 formula has the just about same speed compared with the traditional RC6 one. Nevertheless, the planned formula improves secret writing security by inserting the cruciate layer as a result of a differential Associate in Nursingd linear analysis encompasses a problem in analyzing an encrypted stream.

\subsubsection{Symmetry Layer Structure}

In this paper, the planned symmetry layer is quick by consisting of computer memory unit unit logic operation and stuck rotate operation once enforced via hardware and software system. The half whole RC6 spherical uses secret writing procedure and therefore the remainder of it use secret writing one, and symmetry layer has been place into the center of secret writing and secret writing. so the formula between secret writing and secret writing has become same, and therefore the performance of RC6 has been improved.

The following is that the goals of symmetry layer.

- Having RC6 to own constant thanks to write and rewrite.

- Having the safety of RC6 increase by inserting the symmetry layer.

- ought to be straightforward once enforced via software system and hardware.

- There should be no distinction between the planned formula and original one within the side of the method speed.

The implementation of symmetry layer in RC6

When applying the symmetry layer into RC6 formula, this formula use operations within the original spherical functions while not modification. but it inserts secret writing formula into the half the complete progress rounds, applies 
secret writing formula into the remainder of it, and inserts symmetry layer within the middle of secret writing and secret writing formula.

Figure a pair of shows the complete method of the planned formula.

First of all, secret writing executes ten sphericals secret writing once execution spherical keys and ADD operations as change of color stage before round functions. every spherical operations use 232 bits spherical key to feature operation. within the next, the appliance of the symmetry layer is dead because the clarification of the symmetry layer as shown in Figure one, and uses four 32bits spherical keys. the remainder ten rounds apply the secret writing formula of RC6, and once the execution of figure operation of 2 32bits spherical keys in every spherical and therefore the last change of color method, finally 128 bits cipher-text is made.

The secret writing of the planned formula is dead as

Figure 2, and therefore the application of spherical keys is that the inverse of the method of secret writing. And in change of color stage, the dead ADD operation is switched to figure operation, and therefore the method of symmetry layer is applied because the inverse of the secret writing. In this paper, the key programing of the planned formula uses the RC6 key programing with none modification. due to creation of 32bits four keys utilized in solely symmetry layer, this formula uses total $32 \mathrm{bits}$ forty eight key.

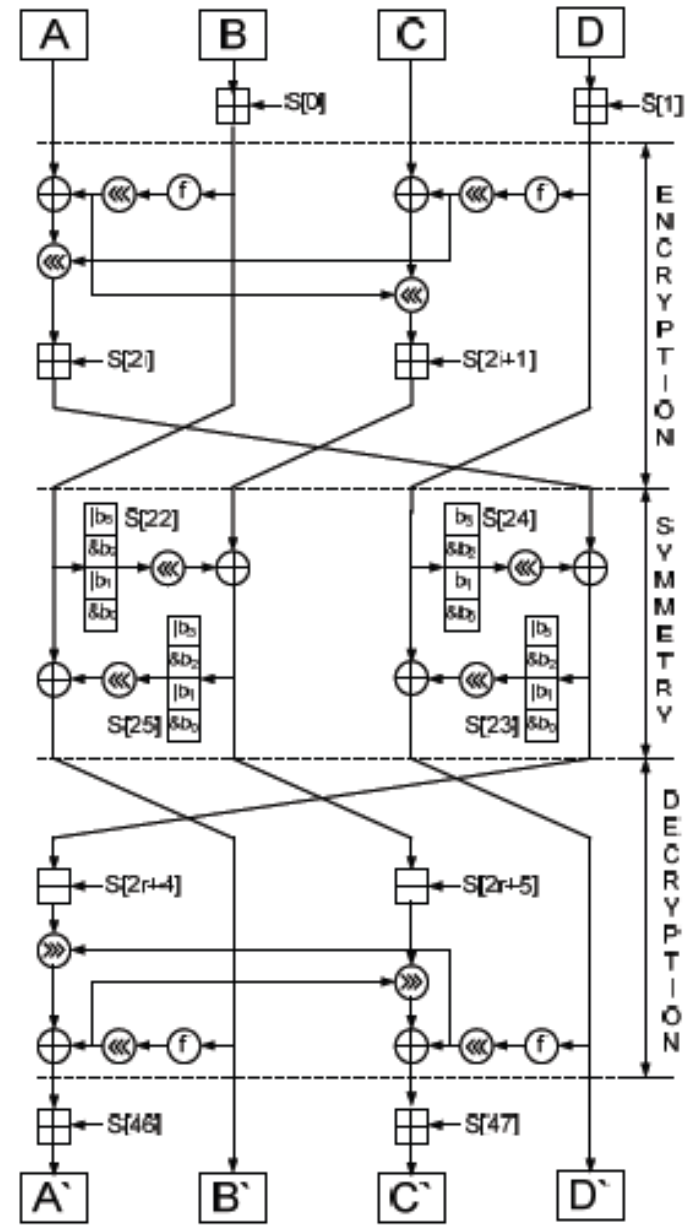

Fig 5: Proposed Methodology for RC6

\section{CONCLUSIONS}

We have designed and enforced TARF, a strong trust-aware routing framework for WSNs, to secure multi-hop routing in dynamic WSNs against harmful attackers exploiting the replay of routing data. TARF focuses on trait and energy potency, that ar very important to the survival of a WSN in a very hostile surroundings. With the concept of trust management, TARF allows a node to stay track of the trait of its neighbors and so to pick out a reliable route. not like previous efforts at secure routing for WSNs, TARF effectively protects WSNs from severe attacks through replaying routing data; it needs neither tight time synchronization nor identified geographic information. The resilience and quantifiability of TARF is evidenced through each intensive simulation and empirical analysis with largescaleWSNs; the analysis involves static and mobile settings, hostile network conditions, further as sturdy attacks like hollow attacks and Sybil attacks.

\section{REFERENCES}

[1] J. Newsome, E. Shi, D. Song, and A. Perrig, Apr 2004 "The sybil attack in sensor networks: Analysis and defenses," in Proc. of the 3rdInternational Conference on Information Processing in Sensor Networks (IPSN'04).

[2] L. Bai, F. Ferrese, K. Ploskina, and S. Biswas,2009 "Performance analysis of mobile agent-based wireless sensor network," 8th International Conference on Reliability, Maintainability andSafety (ICRMS 2009), pp. $16-19$.

[3] L. Zhang, Q. Wang, and X. Shu, 5-7 2009 "A mobileagent-based middleware for wireless sensor networks data fusion," Instrumentation and Measurement Technology Conference (I2MTC'09), pp. 378 -383.

[4] W. Xue, J. Aiguo, and W. Sheng,12-14 2005 "Mobile agent based moving target methods in wireless sensor networks," , IEEE International Symposium on Communications and Information Technology (ISCIT2005), vol. 1, pp. $22-26$.

[5] J. Hee-Jin, N. Choon-Sung, J. Yi-Seok, and S. DongRyeol,2008 "Amobile agent based leach in wireless sensor networks," 10th International Conference on Advanced CommunicationTechnology (ICACT 2008), vol. 1, 17-20 , pp. $75-78$.

[6] J. Al-Karaki and A. Kamal,Dec 2004 "Routing techniques in wireless sensornetworks: a survey," Wireless Communications, vol. 11, no. 6, pp.6-28.

[7] G. Zhan, W. Shi, and J. Deng, 2010“TARF: A trustaware routing framework for wireless sensor networks," $7^{\text {th }}$ European Conference on Wireless Sensor Networks (EWSN'10).

[8] F. Zhao and L. Guibas, "Wireless Sensor Networks: An Information Processing Approach"2004, Morgan Kaufmann Publishers.

[9] A. Wood and J. Stankovic,Oct 2002 "Denial of service in sensor networks," Computer, vol. 35 , no. 10 , pp. 54 62 .

[10] C. Karlof and D. Wagner,2003 "Secure routing in wireless sensor networks: attacks and countermeasures" 1st IEEE International Workshop on Sensor Network Protocols and Applications. 
[11] M. Jain and H. Kandwal,2009 "A survey on complex wormhole attack in wireless ad hoc networks," International Conference on Advances in Computing, Control, and Telecommunication Technologies (ACT '09), pp. $555-558$.
[12] I. Krontiris, T. Giannetsos, and T. Dimitriou, "Launching a sinkhole attack in wireless sensor networks; the intruder side," IEEE International Conference on Wireless and Mobile Computing, Network. 